läßt eine deutliche Änderung der Tropfenzahl auf die Anwesenheit einer Lipase schließen, die gegen Chinin unempfindlich, also blutfremd ist. Die Tropfenzahl unserer Pipette war für reines dest.Wasser 94, für gesättigte Tributyrinlösung etwa I30: Erhöhung der Temperatur um Io erhöht die Tropfenzahl um 2. Um jeden Irrtum auszuschließen, haben wir in einzelnen Fällen die Chininmenge noch weit höher gewählt, bis zu $20 \mathrm{mg}$. Da aber Chinin an sich die Oberflächenspannung erhöht, wird durch so hohe Chinindosen die Reinheit der Versuchsergebnisse etwas getrübt, insbesondere wird die Berechnung der Tributyrinkonzentration aus der Tropfenzahl dadurch erschwert. Wir verzichten daher im folgenden ganz auf diese Umrechnung und teilen nur die gefundene Tropfenzahl mit, aus der das Wesentliche mit genügender Genauigkeit ersehen werden kann. Folgende kleine Tabelle zeigt das Verhalten der im Blute vorhandenen Lipase gegen Chinin.

Tabelle I.

\begin{tabular}{|c|c|c|c|c|c|c|c|c|}
\hline \multirow[t]{2}{*}{ Nr. } & \multirow{2}{*}{ Diagnose } & \multirow{2}{*}{$\begin{array}{l}\mathrm{mg} \text { Chinin } \\
\text { in } 57 \mathrm{ccm}\end{array}$} & \multicolumn{3}{|c|}{ Iropferzahl } & \multirow{2}{*}{\multicolumn{3}{|c|}{ Bemerkungen }} \\
\hline & & & $3^{\prime}$ & $30^{\prime}$ & $60^{\prime}$ & & & \\
\hline \multirow{2}{*}{ I } & Glomerulonephritis & 20 & 130 & I 30 & $\mathrm{I} 28$ & \multirow{2}{*}{\multicolumn{3}{|c|}{$33 \mathrm{ccm}$ Serum }} \\
\hline & & & & & 93 & & & \\
\hline \multirow[t]{2}{*}{2} & Asthma bronch. & 20 & I 29 & I 29 & 128 & & & \\
\hline & & 0 & 122 & 98 & 94 & & , & ", \\
\hline \multirow{2}{*}{3} & \multirow{2}{*}{ Gelenkrheumatismus } & 2 & I 33 & I 33 & I 33 & & & \\
\hline & & o & I35 & I 3 I & 125 & & " & ") \\
\hline \multirow[t]{2}{*}{4} & \multirow[t]{2}{*}{ Endocarditis lenta } & 2 & I35 & I 35 & I 34 & & & \\
\hline & & 0 & I 36 & I 3 I & I 26 & & $"$ & $\because$ \\
\hline \multirow{2}{*}{5} & \multirow{2}{*}{ Schrumpfniere . } & 2 & 126 & I 26 & - & & & \\
\hline & & I & I 26 & I26 & 124 & & "' & , \\
\hline \multirow[t]{2}{*}{6} & \multirow[t]{2}{*}{ Normalfall } & 2 & $12 \mathbf{i}$ & I $2 \mathrm{I}$ & 120 & 3 & & \\
\hline & & 0 & I $2 \mathrm{I}$ & I I 5 & IOI & & " & , \\
\hline
\end{tabular}

In allen 6 Fällen ist die Spaltung des Tributyrins durch das Chinin völlig gehemmt worden, denn die Tropfenzahl hat sich nach einer Stunde nicht geändert, während die ohne Chinin angesetzten Kontrollen alle eine sehr starke Spaltung zeigen. Anders verhält es sich bei Leberkranken.

Tabelle II.

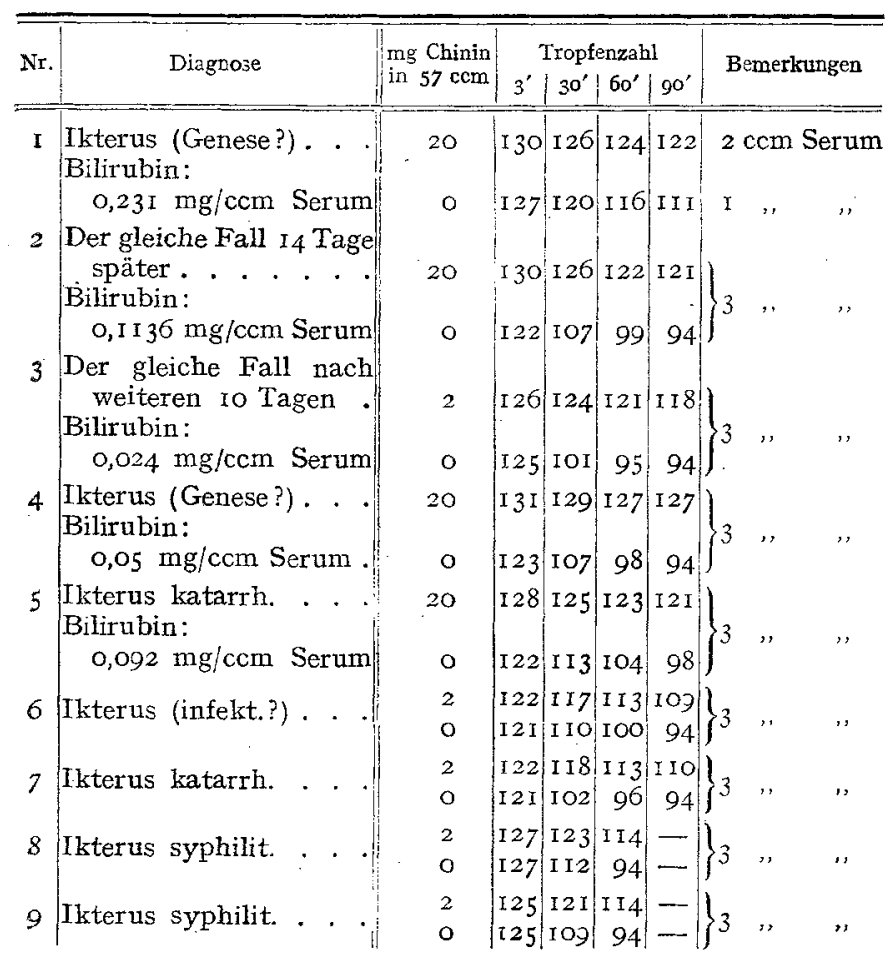

Wie aus dieser Tabelle ersichtlich, ist bei diesen Fällen die Tropfenzahl am Ende des Versuches auch in den mit Chinin versetzten Lösungen wesentlich kleiner, als am Anfang. Es hat also trotz des Giftzusatzes eine Spaltung des Fettes stattgefunden, d. h. es muß eine chininresistente Lipase in den Seren zugegen gewesen sein. Da aber die Spaltung in den Kontrollen in derselben Zeit weitergegangen ist, mu $B$ man annehmen, daß neben der chininresistenten eine chininempfindliche Lipase in den Seren war. Man geht wohl nicht fehl, wenn man diesen Befund dahin deutet, daß neben der normalen Serumlipase Leberlipase im Blute dieser Patienten kreiste. Nur in einem der untersuchten Fälle, Nr. 4, war die Spaltung durch Chinin so weit gehemmt, daB es zweifelhaft bleiben muß, ob hier eine chininresistente Lipase angenommen werden darf. Dieses Serum stammte von einem Patienten, der in der Heilung begriffen war. Die Gallensperre war hier schon durchbrochen und der Bilirubinwert im Blute auf $0,05 \mathrm{mg}$ gesunken.

Die von uns beschriebene Methode dürfte geeignet sein, in zweifelhaften Fällen, bei denen die Frage hepatogener oder hämolytischer Ikterus $\mathrm{zu}$ entscheiden ist, als differentialdiagnostisches Hilfsmittel zu dienen. Aber nicht jede chininfeste Lipase ist als Leberlipase zu deuten. Wir fanden nämlich bei einigen Nephrosen ebenfalls eine, erst durch sehr hohe Chininmengen vergiftbare Lipase im Blute, und da BLocH in einer noch nicht veröffentlichten Arbeit zeigen konnte, daß bei degenerativen Nierenerkrankungen eine Lipase im Urin auftritt, sind wir geneigt, diese pathologische Lipase im Blute als aus der Niere stammend anzusehen. Ob sich diese "Nierenlipase" von der ,Leberlipase“ unterscheiden läßt, bleibt weiteren Studien vorbehalten. Die prinzipielle Möglichkeit dazu ist durchaus gegeben, denn die verschiedenen Lipasen unterscheiden sich nicht nur durch ihr verschiedenes Verhalten gegen Chinin, sondern auch gegen andere Gifte. So ist z. B. die aus dem Pankreas stammende Lipase resistent gegen Atoxyl, während Serumlipase sowohl als auch Leberlipase schon durch kleinste Mengen dieses Giftes völlig gehemmt werden ${ }^{2}$ ). Auch dieses unterschiedliche Verhalten dürfte ein wertvolles diagnostisches Mittel abgeben bei Pankreasaffektionen, die ja unter Umständen nicht leicht zu erkennen sind. Leider konnten wir mangels geeigneter Fälle bisher nicht die "Pankreaslipase" im Blute identifizieren, hoffen aber bald das Versäumte nachholen zu können. Die von uns mitgeteilten Versuche sollen nur das Prinzipielle des Verfahrens zeigen. Es wird noch mancher Arbeit, klinischer und experimenteller Art, bedürfen, ehe wir in der Lage sein werden, alle Möglichkeiten zu übersehen. Immerhin glauben wir, daß hier ein Weg betreten worden ist, der auch für die praktische Medizin zu brauchbaren Ergebnissen führen kann.

Literatur. 1) Vgl. P. Rona und E. BAch, Biochem. Zeitschr. III, I66. 1920. P. RoNa und E. BLoCh, ebenda II8, I85. I92I. P. RoNa and D. ReINicke, ebenda II8, 213. I92I, - 2) P. RoNA und R. Pavlovic, ebenda r30, 225. 1922, - ${ }^{3}$ ) P. Rona und R. PAvLovic, ebenda I33. I922.

\section{DIE PNEUMOKOKKEN IN DER UMGEBUNG GESUNDER UND KRANKER.}

\section{Von}

Professor Dr. A. SEITZ.

Aus dem Hygienischen Institut Leipzig. (Direktor: Geheimrat KRUSE.)

Seit den Untersuchungen von A. FRäNKEL, die zeigten, daß der Erreger der sogenannten Kaninchenseptikämie identisch ist mit dem Pneumococcus lanceolatus der Lungenentzündung, erhob man Befunde, daß der Speichel auch ganz gesunder Personen vielfach virulente Pneumokokken enthält. Es sei hier an die Untersuchungen NETTERS erinnert, der in 20\% seiner Speicheluntersuchungen virulente Pneumokokken nachgewiesen haben will, an v. BESSER u. a., der unter 57 untersuchten Fällen I 4 mal, also in $24 \%$ auch im Bronchialsekret gesunder Personen die Erreger virulent fand, an KRUSE und Pansini, welche sie außerdem nicht selten auch im Urin feststellten. Ein Teil der Forscher folgerte nun aus diesen Befunden, welche gewissermaßen die Ubiquität des Pneumokokkus dartaten, daß die Lungenentzündung überhaupt durch Einwanderung der in der normalen Mundhöhle vorhandenen Keime in die Lungen zustande kommt, unter Mitwirkung des epidemiologischen Lückenbüßers der „,Dis- 
position" - Erkältungen oder ähnliche Ursachen, welche eine Herabsetzung der Widerstandskraft im Gefolge haben, teilweise durch eine temporäre Minderung der normalen Schutzstoffe des Körpers. Daß in der Tat allgemeine und lokale Disposition eine Rolle spielen; gelingt ja auch im Tierversuch nachzuweisen; es sei nur an die bekannten Erkältungsversuche mit nachfolgender künstlicher Infektion erinnert. Diese Vorstellungen haben vielfach Stützen bei klinischen Beobachtern gefunden. So weist ein guter Kenner der Pneumonie, wie AUFRECHT, daraufhin, daß nichts dafür spricht, was bei dieser Krankheit den Verdacht einer Ansteckung nach dem Modus typischer Infektionskrankheiten aufkommen läßt. In der Tat findet eine Übertragung der Krankheit selber des Pneumonikers, auf seine nächste Ungebung, beispielsweise von Bett zu Bett, ja kaum. statt. Aber wenn auch keine Ubertragung der. Krankheit als solcher erfolgt, so wäre es doch von Interesse festzustellen, ob nicht eine Häufung von virulenten Pneumokokken in der Umgebung von Pneumoniekranken stattfindet. Aufschlüsse hierüber können auch ein Bild ergeben über die Verbreitungsweise der Pneumonie überhaupt. Wir stellten hierüber Erhebungen an, welche wir uns gedacht hatten unter Mitwirkung der Ärzteschaft an der Hand eines Fragebogens. Leider blieben diese Untersuchungen in den Familien in der Minderzahl, und das Material wurde daher größtenteils den Leipziger Krankenhäusern entnommen. Wir gingen so vor, daß wir aus dem Auswurf des Pneumonikers durch Mauspassage den Pneumoniestamm isolierten, und zeitlich damit übereinstimmend seine Umgebung untersuchten auf virulente Pneumokokken. Diese Umgebnng besteht aus Pflegern und den nahen Angehörigen, welche ständig mit dem Kranken in Berührung kamen. Von dem Speichel der Umgebung wurde I $\mathrm{ccm}$ gemischt mit I ccm Traubenzuckerbouillon, Mäusen gleichen Gewichts subcutan verimpft. Von den isolierten Pneumokokkenstämmen wurden sofort, um Virulenzabschwächung $z \mathbf{u}$ vermeiden, Serumbouillonröhrchen angelegt, und von der eintägigen Kultur später die genaue Virulenz an Mäusen in üblicher Weise festgestellt.

Die Prüfung des normalen Speichels zunächst ergibt geringere Prozentsätze als NETTER u. a. angeben, sogar wenn man Individuen wählt, die durch ihren Verkehr in Laboratoriumsräumen oder sonstige Tätigkeit in Pneumokokken angereicherten Räumen, Keime durch Dielenstaub usw. aufgenommen haben könnten. Wie ja die Untersuchungen von StILMAN und andere amerikanische Arbeiten der Kriegsjahre ergeben, liegt in der Tat die Ubertragungsmöglichkeit gerade durch Dielenstaub von Krankenzimmern vor. Aber trotz dieser relativen Ubiquität der Pneumokokken sind die Befunde von pathogenen Pneumokokken bei gesunden Menschen mit normaler Umgebung nicht so häufig. Wie ist das nun

Tabelle I: Auswurf von Pneumonikern.

Unter I8. Auswurf-Untersuchurgen 12 mal virulente

Pneumokokken .................... $66 \%$

Unter 18 Auswurf-Untersuchungen 5 mal hämolytische

$$
\text { Streptokokken. . . . . . . . . . . . . . . = }=27,7 \%
$$

\section{Speichel aus der Umgebung won Pneumonikern.} (Pfleger und Angehörige.)

Unter 7I Personen fanden sich I6 mal virulente Pneumokokken ........................... $=22,5 \%$

Unter 71 Personen fanden sich to mal hämolytische

$$
\text { Streptokokken. . : . . . . . . . . . }=14 \%
$$

Speichel anderer Personen.

Unter 53 Personen fanden sich 2 mal virulente Pneumo-

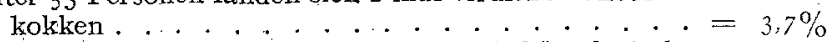
Unter 53 Personen fanden sich 5 mal hämolytische

$$
\text { Streptokokken. . . . . . . . . . . . . . }=9.4 \%
$$

bei der Umgebung von Preumoniekranken? Unter 53 Personen aus Nicht-Pneumonieumgebung fanden sich in $3,7 \%$ virulente Preumokokken, während die Untersuchung der Krankenumgèbung ( 7 I Personen) 22,5\% Keimträger ergab. Dem entsprachen aus dem Auswurf von Pneumonikern selbst isolierte Pneumokokikenstämme 66\%. Wir gewinnen den Eindruck, daß wir es bei der Lungenentzündung mit ähnlichen Verhältnissen zu tun haben wie bei der epidemischen Menin- gitis, d. h. es findet eine Anreicherung der Umgebung des Kranken mit Keimträgern statt. Möglicherweise ist der weitere Verlauf so, daß eine Steigerung der Virulenz durch Passagen von Keimträger auf Keimträger stattfindet, bis unter Hinzutreten besonderer, die Krankheit erleichternder Momente, diese zum Ausbruch kommt. Neufeld hat neuerdings auf die epidemiologischen Beobachtungen der Amerikanel. hingewiesen; besonders die Erfahrungen von CoLE und MCCALLum (Arbeiten a.d. Rockefeller-Institut I920, Bd. 34) zeigen, wie auch drüben die Beobachtung gemacht wird, daß die Lungenentzündung offenbar zum großen Teil auf ektogene Ansteckung zurückzuführen ist. Auffallend ist die Häufung von hämolytischen Streptokokken bei den Pneumonikern selbst und bei den Personen ihrer Umgebung, während die übrige Bakterienflora keine Absonderheiten zu erkennen gibt. Das Rockefeller-Institut fand im Winter $1917 / 18$ in verschiedenen Hospitälern der amerikanischen Armee, seltner unter Zivilisten, daß diese hämolytischen Streptokokken speziell dort $\mathrm{zu}$ finden waren, wo die Lungenentzündungen klinisch atypisch, mit Komplikationen, verliefen, oder sie von Masern vergesellschaftet waren. Ahnliches konnten wir nach Rundfrage an unserem Material nicht feststellen. Wohl aber können wir annehmen, daß die hämolytischen Streptokokken als solche, auch ohne Masern, eine virulenzerhöhende Eigenschaft für Pneumokokken besitzen. Ganz ähnlich scheinen Kinderkrankheiten, Masern, Scharlach, Pertussis, Diphtherie, eine Häufung aufzuweisen dort, wo Erkrankungen und Sterblichkeit an Lungenentzündung in aufsteigender Linie sich bewegen, eine epidemiologische Ubereinstimmung auf die Kruse bereits 19r 3 hinwies. Wenn wir unser Material daraufhin untersuchen, wie die Unterbringungsverhältnisse der Umgebungspersonen beschaffen waren, so ist unverkennbar der Einfluß, den dichtgedrängtes Wohnen auf die Verbreitung der Pneumonie hat. Die Kranken waren teilweise in alten Krankenhäusern mit dichter Belegung und leichter Möglichkeit der Kontagion untergebracht, so daß sich die Zusammendrängung in diesen Krankensälen vergleichen läßt mit der mangelhaften Unterkunft, wie sie die Wohnungsnöte in den von uns untersuchten Familien hervorgerufen hatten. Im Gegensatz hierzu steht ein ganz modernes Krankenhaus St. G., mit den Anforderungen moderner Hygiene genügenden Einrichtungen. Wir möchten es daher nicht als zufällig betrachten, daß sich in letzterem weniger Pneumokokkenträger fanden. wie an den übrigen Orten der Untersuchung.

Tabelle II.

\begin{tabular}{l|c|c|c}
\hline $\begin{array}{c}\text { Ort } \\
\text { der Untersuchung }\end{array}$ & $\begin{array}{c}\text { Zahl } \\
\text { der Patienten }\end{array}$ & $\begin{array}{c}\text { Zahi d. Umgebrngs- } \\
\text { untersichungen }\end{array}$ & $\begin{array}{c}\text { Wie oft virul. Pneumok. } \\
\text { in dor Ungebung }\end{array}$ \\
\hline \hline Priv. . . . & 3 & 8 & $5=62,5 \%$ \\
Khs. . . . & 4 & 2 & $2=100 \%$ \\
St. J. . . & 7 & 31 & $5=16,1 \%$ \\
St. G. . . & 4 & 30 & $4=13,3 \%$ \\
\hline & I8 & 71 & I6
\end{tabular}

Biologisch ließen sich zwischen den Pneumokokkenstämmen aus Patienten und aus Keimträgern keine wesentlichen Differenzen feststellen. Die Neigung, lange Ketten zu bilden, war jedoch bei den schwach virulenten Stämmen ausgeprägt; durch Verdauungsversuche ließen sich die Stämme nicht oder kaum beeinflussen, ob sie virulent waren oder nicht. Anders allerdings bei der Neufeldschen Verdauungsprobe mit Galle, resp. taurocholsaurem Natrium; während die virulenten Pneumokokkenstämme (Bouillonkultur) eine restlose Auflösung schon nach kurzer Zeit ergaben, lösten sich dicjenigen Stämme, die aus Keimträgern isoliert worden waren, entweder gar nicht oder zeigten nur eine Aufhellung. Die Prüfung der Virulenz die möglichst sofort angestellt wurde, stets ahne Passage über feste Nährboden, ergab folgendes (Ausschnitt aus den Versuchsprotokollen):

Die aus Patienten isolierten Stämme hatten also durchschnittlich eine Virulenz von 1/1000 Öse, teils von 1/100 Ösé, seltener von $1 / 10$ Ose (stets flüssiger Kultur). Dem entsprachen Virulenzen der entsprechenden Stämme aus der Umgebung dieser Patienten von durclischnittlich I Ose, seltener $1 / 10$ Ose; 
Tabelle III.

\begin{tabular}{|c|c|c|c|c|c|c|c|c|c|c|c|c|c|c|}
\hline \multirow{2}{*}{$\begin{array}{c}\text { Patient.- } \\
\text { Name } \\
\text { u. }-\mathrm{Nr} . \\
\end{array}$} & \multirow{2}{*}{\multicolumn{2}{|c|}{ Patienten }} & \multirow{2}{*}{\multicolumn{3}{|c|}{$\begin{array}{c}\text { Virulenz } z \\
\text { im Mäuseversuch }\end{array}$}} & \multicolumn{4}{|c|}{ Patientenaus Umgebung } & \multirow{2}{*}{$\begin{array}{l}\text { Patienten- } \\
\text { Name u. -Nr. }\end{array}$} & \multirow{2}{*}{ Datum } & \multirow{2}{*}{\multicolumn{3}{|c|}{$\begin{array}{l}\text { Normaler Speichel } \\
\text { Virulenz im Mäuseversuch }\end{array}$}} \\
\hline & & & & & & $\begin{array}{l}\text { Name } \\
\text { resp.Nr. }\end{array}$ & Datum & Herkunft & im ,Mäusseversuch & & & & & \\
\hline I B. & 12. I. 22 & St. G. Sta. $2^{\mathrm{II}}$ & $1 / 1000$ & Öse & $\dagger$ & 46 & I2. I. 22 & St. G. Sta. $2^{\text {II }}$ & $1 / 10$ Öse $\dagger$ & 3 (Dr. K.) & $24 \cdot 2.22$ & $1 / 10$ & Óse & $\begin{array}{l}\text { überlebt } \\
\dagger\end{array}$ \\
\hline $2 \mathrm{~F}$. & 12.1 .22 & St. G. Sta. $2^{\mathrm{KI}}$ & $1 / 100$ & ", & $\dagger$ & 68 & $26.7 \cdot 22$ & St. G. Sta. 2 II & , $\quad \dagger$ & 23 (Pohle) & 26.6 .22 & $1 / 10$ & , & $\begin{array}{c}\text { überlebt } \\
\dagger\end{array}$ \\
\hline $3 P$. & I2. I. 22 & St. G. Sta. $2^{\pi}$ & $1 / 1000$ & $\therefore$ & $\dagger$ & 69 & $26.7 \cdot 22$ & St. G. Sta. $2^{\mathrm{II}}$ & , $\dagger$ & 27 (Öhser) & 15.6 .22 & $1 / 10$ & , & $t$ \\
\hline $4 \mathrm{H}$. & 12.1 .22 & St. G. Sta. $2^{\mathrm{II}}$ & $1 / 1000$ & , & $\dagger$ & 70 & 26.7 .22 & St. G. Sta. 2II & , & 29 (Hesse) & $3 \cdot 7 \cdot 22$ & $\begin{array}{c}1 / 10 \\
1\end{array}$ & $"$, & $\begin{array}{c}\text { überlebt } \\
\quad,\end{array}$ \\
\hline $5 B$. & 30.1 .22 & St. Jac. B. I9 & $1 / 1000$ & " & $\dagger$ & ${ }_{53}^{52}$ milie & $\begin{array}{l}7.2 .22 \\
7.2 .22\end{array}$ & Familie & $\begin{array}{cc}1 / 10 & \text { Ose lebt } \\
\text { u. I Ose } t \\
1 / 10 \text { Ose } t\end{array}$ & 44 (Nickel) & 27.6 .22 & $\begin{array}{c}1 / 10 \\
1\end{array}$ & ", & ", \\
\hline $6 \mathrm{R}$. & 10.2 .22 & $\begin{array}{l}\text { Kinderkr. } \\
\text { Stat. IO4 }\end{array}$ & $1 / 10$ & $\because$ & + & 57 & 19.2 .22 & $\begin{array}{l}\text { Kinderkr. } \\
\text { Stat. IO4 }\end{array}$ & $1 / 20 \quad, \quad \dagger$ & & & & & \\
\hline $7 \mathrm{~L}$. & 16.2 .22 & $\begin{array}{l}\text { Kinderkr. } \\
\text { Stat. IO4 }\end{array}$ & $1 / 10$ & ," & $\uparrow$ & 54 & 10.2 .22 & Kinderkr. & $1 / 10 \quad, \quad \dagger$ & & & & & \\
\hline $8 \mathrm{~A}$. & 24.2 .22 & $\begin{array}{l}\text { Kinderkr. } \\
\text { Stat. IO4 }\end{array}$ & $1 / 10$ & " & $\dagger$ & 59 & $20.5 \cdot 22$ & $\begin{array}{l}\text { Kinderkr. } \\
\text { Stat. } \mathrm{IO}_{4}\end{array}$ & $\mathbf{I} \quad$ is $\dagger$ & & & & & \\
\hline $9 \mathrm{G}$ & 22.2 .22 & St. Jac. B. I9 & $1 / 100$ & ", & + & 20 & $7 \cdot 1.22$ & St. Jac. B. I9 & $\begin{array}{l}\text { T/10 Öse } \\
\text { u. I Ose lebt }\end{array}$ & & & & & \\
\hline Io Pf. & $22 \cdot 5 \cdot 22$ & St. Jac. B. I7 & $1 / 1000$ & ", & $\dagger$ & $2 \mathrm{I}$ & & & u. I Ose lebt & & & & & \\
\hline I I $R$. & $24 \cdot 5 \cdot 22$ & St. Jac. B. ro 4 & $1 / 1000$ & ", & $\uparrow$ & 17 & $\begin{array}{l}7.1 .22 \\
27.5 .22\end{array}$ & $\begin{array}{l}\text { St. Jac. B. I9 } \\
\text { St. Jac. B. I7 }\end{array}$ & $\mid \begin{array}{ccc}1 / 10 & \text { Ose } & \dagger \\
1 / 10 & , & \dagger\end{array}$ & & & & & \\
\hline $12 K$. & $7 \cdot 7 \cdot 22$ & Privat & $1 / 10$ & $"$ & & & & & & & & & & \\
\hline
\end{tabular}

\begin{tabular}{|c|c|c|c|}
\hline \multicolumn{4}{|c|}{ Patienten aus Umgebung } \\
\hline $\begin{array}{l}\text { Name } \\
\text { resp. Nr. }\end{array}$ & Datum & Herkunft & Virulenz im Mäuseversuch \\
\hline I4 & 7. I. 22 & St. Jac. B. Io & $1 / 10$ u. I Ose lebt \\
\hline 16 & 7. I. 22 & St. Jac. B. IO & $1 / 10$ u. I Ose lebt \\
\hline 34 & 12. 1. 22 & St. G. Stat. i & $1 / 10$ Öse $\dagger$ \\
\hline 42 & IT. I. 22 & St. G. Stat. I & $1 / 10,,+$ \\
\hline 59 & 27.2 .22 & Familie &,$\quad \uparrow$ \\
\hline 60 & $20.5 \cdot 22$ & KinderheimLeutzsch & 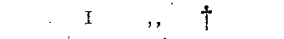 \\
\hline $6 I$ & $20.5 \cdot 22$ & Familie &,$\quad \dagger$ \\
\hline 62 & $20.5 \cdot 22$ & Familie & $1 / 10 \Rightarrow \dagger$ \\
\hline
\end{tabular}

sie näherten sich also dem Grade der. Virulenz der aus normalem Speichel isolierten Pneumokokken.

Anders jedoch, wenn man die Stämme ausagglutinierte. Hierzu stand uns agglutinierendes Pneumokokkenserum aus dem Kochschen Institut zur Verfügung, welches aus Amerika stammte. Diese Sera entsprachen den drei Typen: I. dem von NEUFELD und HäNDEL aufgestellten, meist verbreiteten Typ. I. 2. Dem Repräsentanten der atypischen Stämme, Typ II. 3. Dem Streptoc. mucosus entsprechenden Typ. III.

Bei dieser serologischen Typendiagnose nun ergab sich ein ziemliches Parallelgehen in der Agglutination zwischen den
Patientenstämmen und der Umgebung, gelegentlich ein geringes Ubergreifen auf den anderen Typus. Bei Darstellung dieser Typendiagnose auch mit den ,,normalen" Stämmen, d. h. nicht aus Patientenumgebung isolierten virulenten Pneumokokken, ergibt sich das Vorwiegen des Typ. III.

Der Schutzversuch konnte aus äußeren Gründen noch nicht angestellt werden. Immerhin scheint es denn doch, daB auch bei dieser serologischen Typendiagnose epidemiologische $Z u$ sammenhänge sich ergeben zwischen den Keimträgern aus der nächsten Patientenumgebung (also „,Keimträger im engeren Sinne") und den Pneumoniepatienten selber.

Aus dem epidemiologischen Verhalten der Lungenentzündung, welche mit ihren Keimträgern also große Ähnlichkeit mit anderen Infektionskrankheiten zeigt (Meningitis epidemica z. B.), nun die Berechtigung weitgehender Maßnahmen ableiten $\mathrm{zu}$ wollen, erscheint nicht angezeigt.

Die Erfahrungen, welche z. B. bei der epidemischen Genickstarre in Oberschlesien und Ruhrgebiet mit den übertriebenen Fahndungen nach Keimträgern gemacht worden sind, selbst wenn uns das Gesetz eine wirksamere Waffe in die Hand geben würde, ermuntern nicht zur Nacheiferung. Anders ist ès mit der Aufklörung in breiten Schichten bezüglich des Umgangs mit Auswurf, Wäsche usw. von Pneumo. nikern und demjenigen, was Fi UEGGE treffend als die , Kultur-

Tabelle IV.

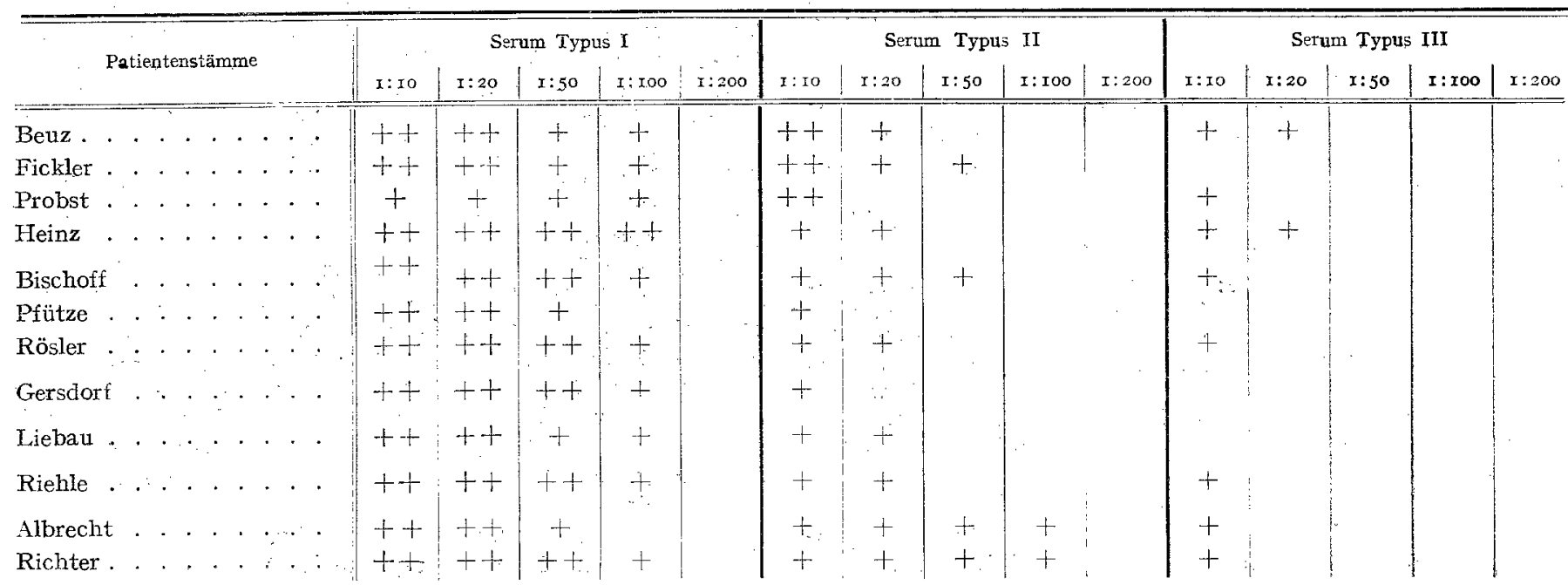




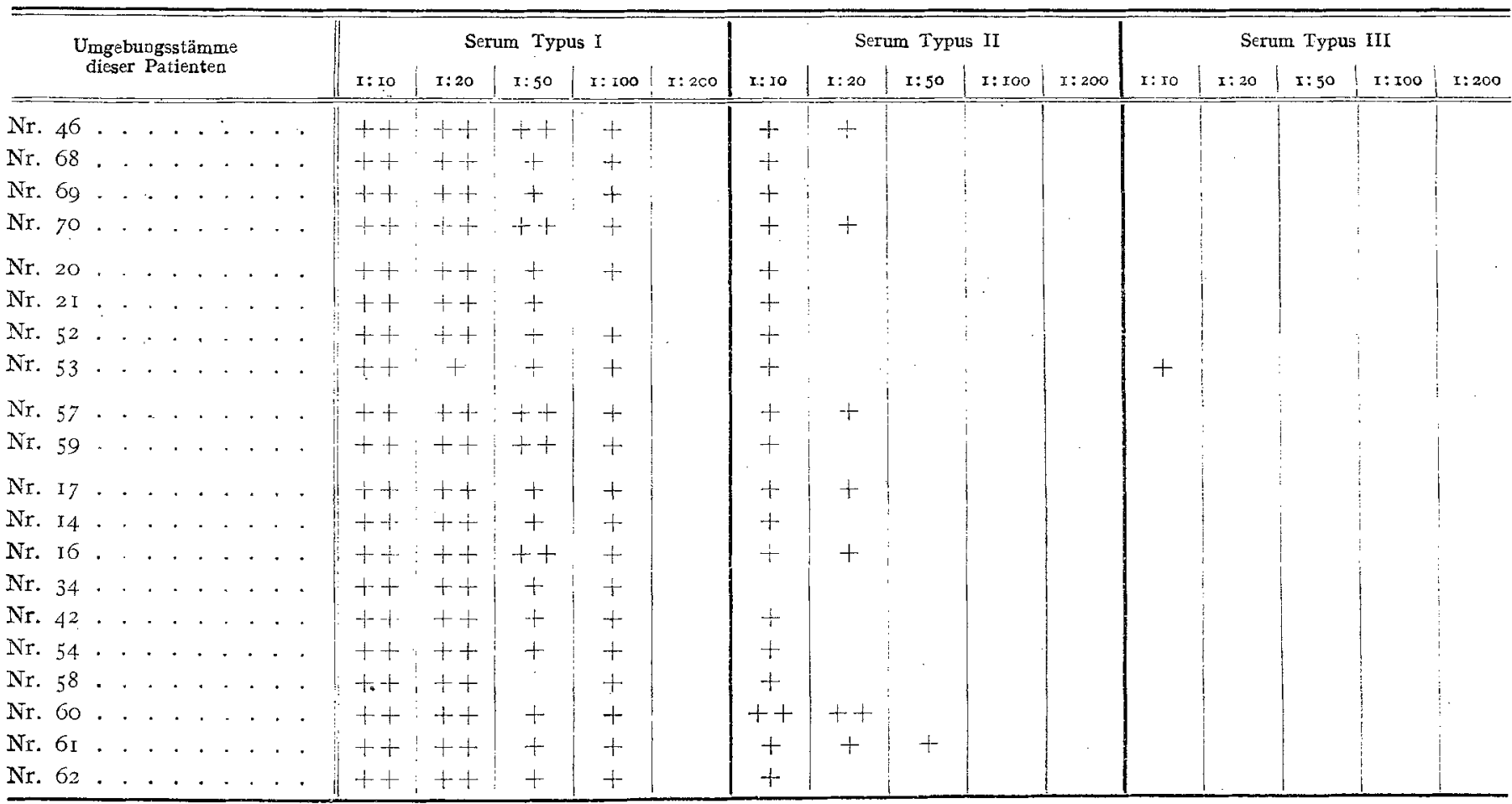

Normale Stämme

Pneumokokkenträger

Nr. 3 (Dr. K.).

Nr. 23 (Pohle)

Nr. 27 (Öhser)

Nr. 29 (Hesse).

des persönlichen Nahverkehrs" (Vermeidung des Anhustens zum Baispiel) bezeichnet hat. Hier möchte auch bei, der Lungenentzündung eine Quelle der Weiterverbreitung liegen, welche durch Aufklärung zum mindesten stark eingedämmt werden kann.

\section{REAKTIVE HYPOGLYKÄMIE DURCH PAREN- TERALE ZUCKERZUFUHR.}

Von

Dr. Rubino und Dr. Varela, Montevideo.

Aus der I. Inneren Abteilung des Städt. Krarkenhauses Charlottenburg-Westend. (Direktor: Prof. UMBER.)

In einer ersten Mitteilung ${ }^{1}$ ) haben wir bereits über die von uns beobachteten Resultate bei Versuchen mit der Rectalzufuhr von konzentrierter Dextroselösung (über 40\%) berichtet. Das wichtigste Ergebnis war die Feststellung des Vorhandenseins von Glykosurien, die durch die Erhöhung des Blutzuckerspiegels nicht erklärt werden konnten, weil dieser stets im Rahmen der physiologischen Grenzen dabei blieb $(0,080$ bis 0, I20 g).

Wir haben diese Versuche unter Anwendung von verdünnter Dextrose-, Lävulose- und Galaktoselösungen (20 bis Ioo $\mathrm{g}$ Zucker), die um Konzentrationen von $3-25 \%$ schwankten, weitergeführt.

Alle unsere Versuche wurden an normalen Individuen angestellt.

Die Technik war folgende: Eine Stunde vor Beginn der Ver* suche wurde ein Reinigungseinlauf gegeben. Die Einführung der Zuckerlösung, die als Tropflilysma erfolgte, dauerte von einer bis zu acht Stunden. Während des Versuches blieb der Patient nüchtern im Bett. Die Veränderungen des Blutzuckerspiegels wurden nach der Bangschen Methode verfolgt. In einigen Fällen haben wir gleichzeitig Parallelbestimmungen der Zahl der roten und weißen Blotkörperchen angestellt.

Die bei dieser Technik gewonnenen Glykämiekurven geben daher ein reines Bild des Blutzuckers und seiner Regulationsvorrichtungen, ohne daß der in minimalen Mengen

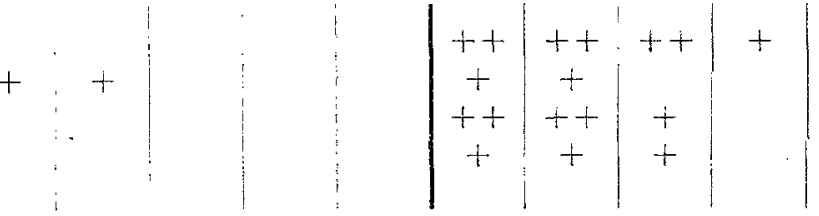

injizierte körperfremde Zucker die Blutzuckerwerte nennenswert beeinflussen kann.

Es sei dabei erinnert, daß die Rectalzufuhr eine zum großen Teil parenterale darstellt, da der AbfluB der unteren Hämorrhoidalvenen unter Umgehung der Leber direkt in die Vena cava inferior erfolgt.

\section{Dextrose.}

Die folgende Tabelle gibt die Resultate wieder, die in $x_{7}$ Dextroseversuchen erzielt wurden.

Die langsame, sehr ausgedehnte Zuckerzufuhr ruft bedeutende Schwankungen des Blutzuckerspiegels hervor, die auch dann noch lange Zeit andauern, wenn die Zuckerzufuhr bereits aufgehört hat. In den meisten Fällen war am Schlusse der Versuche (nach $3 \frac{1}{2}$ bis 6 Stunden) der anfängliche Blutzuckerwert noch nicht wieder erreicht.

Das wichtigste Ergebnis besteht darin, daß in $I_{4}$ voll I 7 Fällen der Blutzuckerspiegel sinkt. Diese Hypoglykämie setzt gleich zu Beginn des Versuches ein und erstreckt sich dann über mehrere Stunden. Daß sie tatsächlich eine Folge der einverleibten Dextrose ist und nicht mit dem nüchternen Zustand der Versuchsperson oder nur der Flüssigkeitszufuhr zusammenhängt, zeigen uns drei (nicht in der Tabelle angeführte) Kontrollversuche an Personen, die unter den übrigen gleichen Versuchsanordnungen lediglich physiologische Kochsalzeinläufe erhielten. In diesen Fällen trat überhaupt keine Schwankung des Blutzuckerspiegels ein.

Uber das Verhalten des Blutzuckerspiegels bei rectaler Dextrosezufuhr ist bisher, außer unserer früheren Publikation, noch nichts bekannt. Es ist bisher vielmehr stets, auch bei peroraler Zufuhr von kleinen Dextrosemengen (ro-2o g) ein Ansteigen der Blutzuckerwerte beobachtet worden [STRAUB ${ }^{2}$ )].

Die von uns beobachtete Hypoglykämie muß daher in engem Zusammenhange mit unserer speziellen Versuchsanordnung stehen. Die wichtigsten Punkte derselben scheinen uns zu sein:

r. die parenterale Zufuhr,

2. die sehr langsame Einführung. 